\title{
El Museo de Galera, dinamizador cultural y económico en el Altiplano
}

Galera, un municipio de 1.200 habitantes situado en el norte de la provincia de Granada, es conocido a nivel nacional e internacional por el importante legado arqueológico que atesora. En su término municipal existen más de 45 puntos arqueológicos catalogados

Las noticias sobre hallazgos arqueológicos en Galera datan del siglo XIX (BARRIL, 1993; FITA, 1916), pero no será hasta 1920 cuando Juan Cabré y Federico de Motos publican la memoria de las excavaciones realizadas entre 1916 y 1918 en la necrópolis de Tútugi (CABRÉ; MOTOS, 1920), cuando Galera pasó a formar una parte importante de la historiografía arqueológica española. Después, en la década de los 60, el arqueólogo alemán Wilhelm Schüle junto con Manuel Pellicer realiza varias campañas de excavación en el Cerro del Real (PELLICER; SCHÜLE, 1962, 1964 y 1966). A partir de los años 80 se comienzan los trabajos en los yacimientos argáricos de CasteIlón Alto y Fuente Amarga por parte del Departamento de Prehistoria de la Universidad de Granada (MOLINA et ál., 1986; CONTRERAS et ál. 1997; FRESNEDA et ál., 1999; RODRíGUEZ-ARIZA et ál., 1999), continuándose los trabajos en el Castellón Alto en los primeros años del siglo XXI (MOLINA et ál., 2004). En el año 2000 comienzan las investigaciones en la necrópolis de Tútugi por parte del Centro Andaluz de Arqueología Ibérica (RODRÍGUEZ-ARIZA et ál., 2004; RODRíGUEZ-ARIZA et ál., 2008; RODRíGUEZ-ARIZA, 2009 y 2010).

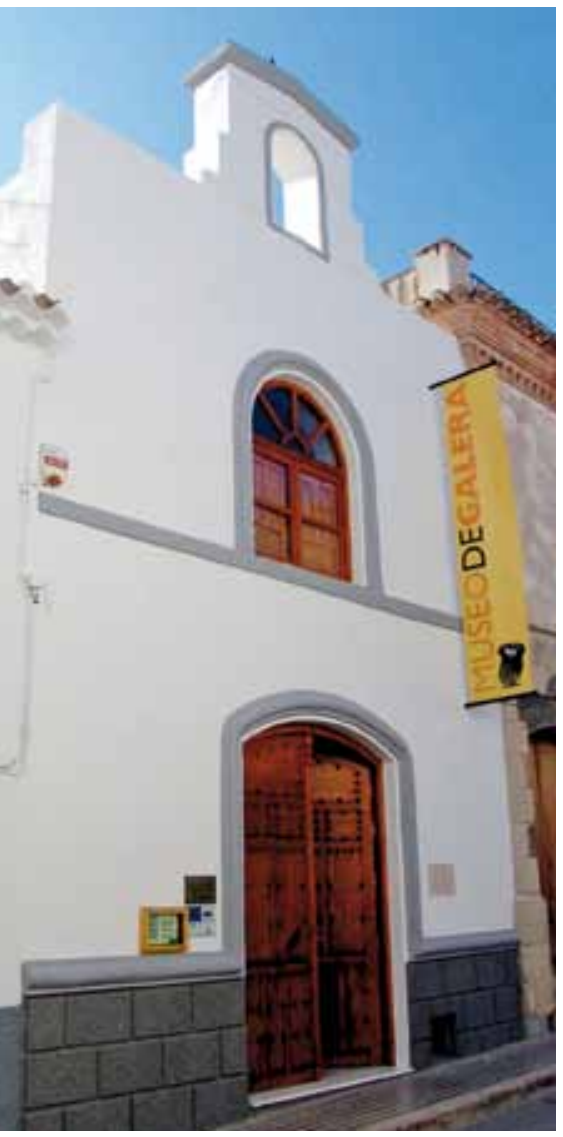

Fachada del museo. Foto: José M. Guillén Ruiz
En este contexto, en el que la arqueología está muy presente, muchos de los vecinos de la localidad conservaban toda una serie de materiales arqueológicos recuperados durante las labores de cultivo de sus tierras. La existencia de estas colecciones privadas fue lo que animó a la creación del museo de Galera.

El museo comienza su andadura en 1998 cuando se comienza a catalogar y restaurar los materiales arqueológicos donados que se encontraban en manos privadas en el pueblo.

Su apertura al público se produce en julio de 2001, aunque en ese primer momento sólo cuenta con dos salas abiertas que conforman la sección de arqueología y no es hasta 2004 cuando se inaugura la sección etnográfica (RODRÍGUEZ-ARIZA; GUILLÉN, 2005).

En 2005 tuvo lugar la remodelación más importante que ha sufrido el museo en sus salas, cuando se decidió la instalación alli de los restos parcialmente momificados de la sepultura 121 de Castellón Alto. La sepultura contiene los restos de dos individuos: un adulto y un niño. Su excepcional estado de conservación ha permitido documentar aspectos hasta ahora desconocidos como son el tipo de peinado, el uso de la lana tricotada, el avanzado trabajo de la madera o la forma de enmangue de una azuela. En un proceso supervisado por el Instituto Andaluz del Patrimonio Histórico se acordó conservar y exhibir los restos en un contenedor que reprodujera con la máxima fidelidad las condiciones que habian propiciado la conservación, para ello se construyó una vitrina con una atmósfera de gas inerte, en la que al introducir nitrógeno puro se extrajo el oxígeno (RODRIGGUEZ-ARIZA; GUILLÉN, 2007).

\section{El conjunto patrimonial de Galera}

El museo de Galera no se ha concebido como una recopilación de materiales expuestos en vitrinas o estanterías, sino que muestra la evolución histórica de la zona. Se inserta a la sociedad humana dentro de su contexto general biogeográfico y geológico, explicando cómo ésta ha ido interactuando con los condicionantes medioambientales, y como ha ido transformando el paisaje. Asimismo, se hace especial mención a las relaciones que las comunidades humanas han establecido entre si en cada momento histórico. Por último, se hace una descripción de la vida cotidiana de estas comunidades, acercándonos a sus trabajos, fiestas, ritos, comidas, creencias, etc.

También hemos pretendido que el museo no se quede aislado, sino insertarlo en la realidad actual de Galera. Para ello, proponemos al visitante realizar algunas de las rutas señalizadas que existen en el municipio, la visita a los yacimientos abiertos al público y que forman parte de la Red de Espacios Culturales de Andalucia o la degustación de la gastronomía de la zona.

En la sala dedicada a la prehistoria reciente existen continuas referencias a los yacimientos argáricos

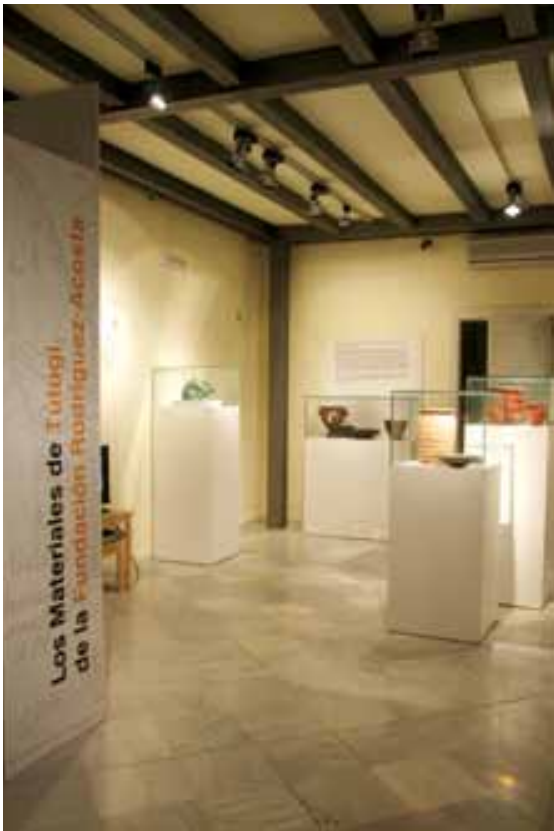

Exposición temporal "Los materiales de Tútugi de la fundación Rodríguez-Acosta".

Foto: Jose M. ํ Guillén Ruiz

excavados en el municipio: Castellón Alto y Fuente Amarga. El primero de ellos, abierto al público, se enmarca dentro de la cultura argárica, en un momento avanzado del Bronce Pleno. Se trata de un poblado agrícola de mediano tamaño en el que el visitante puede conocer cómo era la vida cotidiana en época argárica debido al buen estado de conservación del urbanismo y a la restauración y recreación de una serie de sepulturas y espacios domésticos.

En la sala que abarca los periodos desde el Bronce Final a la Edad Media, el mayor protagonismo lo ostenta el espacio dedicado a la cultura ibérica en el que la gran mayoria de materiales exhibidos proceden del segundo de los enclaves visitables: la necrópolis íbera de Tútugi. En este yacimiento de gran monumentalidad, el visitante puede hacer un recorrido por una de sus zonas con más densidad de enterramientos, comprender cuales eran las técnicas constructivas de esas sepulturas, como enterraban los Iberos a sus difuntos o qué aspecto presentaba una necrópolis en el momento en que ésta estuvo en uso (siglos V a. de C. hasta III a. de C.).

\section{El museo de Galera como influencia en su entorno}

En sus diez años de existencia, el museo de Galera ha influido positivamente en su entorno. Aparte de las funciones propias con respecto al patrimonio arqueológico y etnográfico: investigar conservar y difundir, ha influido en:

- Recuperar el patrimonio mueble que procedente de hallazgos fortuitos estaba en manos de vecinos de Galera. Esta importante relación de objetos ha sido clasificada, restaurada y expuesta para el disfrute y conocimiento de toda la población. 
- Concienciar a la población de la importancia de su patrimonio y del daño que se realiza al saquearlo. Así, desde la creación del museo, el expolio en yacimientos arqueológicos del municipio ha desaparecido prácticamente.

- Afianzar y dar forma a un fuerte sentimiento de identidad de los habitantes de Galera. El museo, como centro donde se explica y recopila toda la información y materiales de los distintos yacimientos arqueológicos, y se recogen distintas actividades etnológicas, se ha convertido en un referente de lo galerino.

- El ser un foco cultural, donde podamos conocer y reflexionar sobre la historia y sus procesos, a través de su exposición permanente, materiales didácticos y actividades y permita también a los visitantes conocer los materiales que, procedentes de Galera, forman parte de las colecciones de otras instituciones, como es el caso de la última exposición temporal, abierta al público en el verano de 2011, en la que

\section{En 2005 tuvo lugar}

la remodelación más importante que ha sufrido

el museo en sus salas,

cuando se decidió la

instalación alli de los restos

\section{parcialmente momificados}

de la sepultura 121 de

Castellón Alto

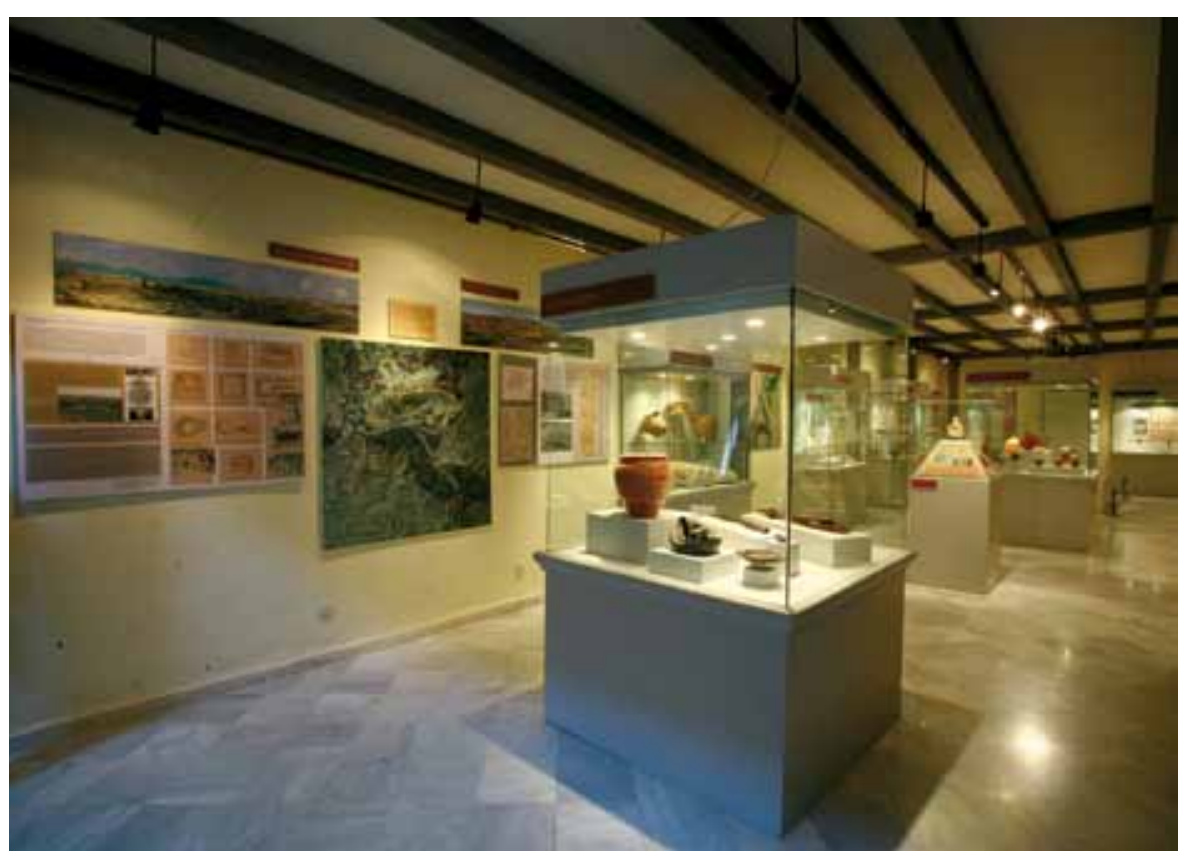

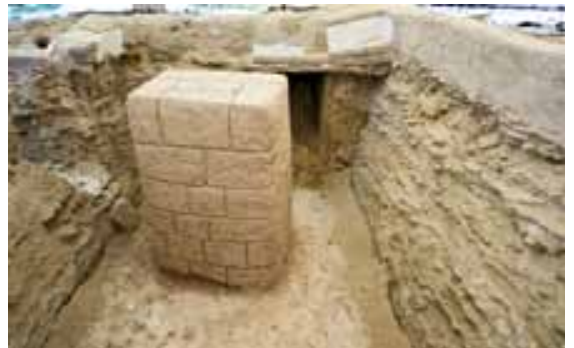

Necrópolis de Tútugi. Foto: Fondo Gráfico IAPH Uuan Carlos Cazalla)

se exhibian los materiales de Tútugi de la fundación Rodriguez-Acosta de Granada.

- Junto a los indudables beneficios culturales, educativos y sociales han de unirse los económicos.

En este sentido no sólo hay que tener en cuenta el impacto en forma de empleos directos, si no la dinámica económica que se genera al convertirse el patrimonio arqueológico de Galera en un factor de atracción de visitantes y en un complemento perfecto para toda la red de alojamientos rurales del Altiplano granadino.

En julio de 2011 el museo de Galera ha cumplido 10 años de existencia. Una vez consolidado como institución de referencia en el norte de la provincia de Granada, hay que poner la vista en la continuación de su labor mediante la apuesta por el patrimonio como uno de los factores de desarrollo claves en uno de los territorios más deprimidos de Andalucía.

José M. ${ }^{a}$ Guillén Ruiz

Coordinador R.E.C.A. Granada

M. ${ }^{a}$ Oliva Rodríguez-Ariza

Directora del museo de Galera, U. de Jaén

\section{Bibliografía}

BARRIL VICENTE, M. (1993) Colección Marqués de Cerralbo. En De Gabinete a Museo. Tres siglos de Historia. Madrid: Museo Arqueológico Nacional, 1993, pp. 406-413 CABRÉ, J.; MOTOS, F. (1920) La Necrópolis ibérica de Tútugi (Galera, Provincia de Granada). J.S.E.A. 25, Madrid, 1920

CONTRERAS, F.; RODRÍGUEZ-ARIZA, M. 0.; CÁMARA, J. A. et ál. (1997) Hace 4000 años... Vida y muerte en dos poblados de la Alta Andalucia. Catálogo de la exposición. Granada: Junta de Andalucia, Fundación Caja de Granada, 1997

FITA, F. (1916) Inscripciones romanas de Chirivel y Galera. En Boletin de la Real Academia de la Historia, LXIX, 1916 pp. 495-498

FRESNEDA, E.; RODRÍGUEZ-ARIZA, M. 0.; LÓPEZ, M. et ál. (1999) El asentamiento argárico de Fuente Amarga (Galera, Granada). XXIV Congreso Nacional de Arqueología, Cartagena 1997. Vol. II. Murcia, 1999, pp. 231-240 MOLINA, F.; AGUAYO, P.; FRESNEDA, E. et ál. (1986) Nuevas investigaciones en yacimientos de la edad del Bronce de Granada. En Homenaje a Luis Siret (1934-1984). Sevilla, 1986, pp. 353-360

MOLINA, F.; RODRÍGUEZ-ARIZA, M. 0.; HARO, M. et ál. (2004) Actuaciones Arqueológicas en el yacimiento de Castellón Alto (Galera, Granada). Campaña de 2001 Anuario Arqueológico de Andalucía 2001. III-1. Sevilla 2004, pp. 435-443

PELLICER, M.; SCHÜLE, W. (1962) Cerro del Real (Galera). Excavaciones Arqueológicas en España 12. Madrid, 1962 PELLICER, M.; SCHÜLE, W. (1966) Excavaciones en el Cerro del Real (Galera, Granada). Excavaciones Arqueológicas en España 52. Madrid, 1966 PEREIRA, J.; CHAPA, T.; MADRIGAL, A. et ál. (ed.) (2004) La necrópolis ibérica de Galera (Granada): la colección del Museo Arqueológico Nacional. Madrid: Museo Arqueológico Nacional, 2004 RODRÍGUEZ-ARIZA, M. 0.; FRESNEDA, E.; PEÑA, J. M. et ál. (1999) Los niveles ibéricos de Fuente Amarga (Galera, Granada). XXIV Congreso Nacional de Arqueología, Cartagena 1997. Vol. III. Murcia, 1999, pp. 283-291 RODRÍGUEZ-ARIZA, M. 0.; MOLINA, F.; TURATTI, R. (2004) Trabajos de documentación arqueológica en la Necrópolis ibérica de Tútugi (Galera, Granada). Anuario Arqueológico de Andalucía 2001. Vol. I. Sevilla, 2004, pp. 144-150

RODRÍGUEZ-ARIZA, M. 0.; GUILLÉN, J. M. (2005) EI

Museo de Galera. mus- $A, 5,2005$, pp. 50-54 RODRIGUEZ-ARIZA, M. 0.; GUILLÉN RUIZ, J. M. (2007) Museo de Galera. Guía Oficial. Granada: Diputación de Granada, 2007

RODRÍGUEZ-ARIZA, M. 0.; GÓMEZ CABEZA, F.; MONTES MOYA, E. (2008) El Túmulo 20 de la Necrópolis ibérica de Tútugi (Galera, Granada). Trabajos de Prehistoria, 65, n. ${ }^{\circ}$ pp. 65-78

RODRíGUEZ-ARIZA, M. 0. (2009) Tútugi. Nuevos trabajos. En ADROHER, A. M.; BLÁNQUEZ, J. Primer Congreso Internacional de Arqueología Ibérica Bastetana Serie Varia 9. Madrid: Universidad de Granada, Universidad Autónoma de Madrid, 2009, pp. 317-334 RODRIGUEZ-ARIZA, M. 0. (2010) Tútugi: del sueño a la realidad. En RODERO, A.; BARRIL, M. (coord.) Viejos yacimientos. Nuevas interpretaciones. Madrid: Museo Arqueológico Nacional-Ministerio de Cultura, 2010, pp $13-52$

Vista sala planta baja del museo. Foto: Miguel Ángel Blanco de la Rubia 